\title{
CARACTERIZAÇÃO FÍSICA DE FRUTOS DE PEQUIZEIRO (Caryocar brasiliense Camb.) DO CERRADO ${ }^{1}$
}

\author{
Nara Fernandes Moura², Lázaro José Chaves³ e Ronaldo Veloso Naves ${ }^{3}$
}

\begin{abstract}
RESUMO - O presente trabalho teve como objetivo caracterizar fisicamente frutos de pequizeiros provenientes do Cerrado para subsidiar estratégias de prospecção, preservação e utilização da variabilidade genética da espécie. Foram coletados frutos de plantas de pequizeiro em duas safras consecutivas (2007/2008 e 2008/2009) em oito regiões do Cerrado. A partir dos frutos coletados procedeu-se a caracterização física dos frutos e os dados foram submetidos à análise descritiva, análise de variância e correlação entre caracteres. Os resultados deste trabalho evidenciaram uma elevada variabilidade fenotípica para a maioria dos caracteres físicos de frutos de pequizeiro nas regiões amostradas. Considerando que parte dessa variabilidade seja de natureza genética, isto indica potencial de ganho genético por meio da seleção. A análise de correlação entre todas as variáveis avaliadas em frutos demonstrou correlações positivas e significativas entre os caracteres de importância para o melhoramento genético da espécie. Existe uma grande diferença entre regiões quanto ao potencial de fornecimento de frutos adequados ao mercado.
\end{abstract}

Palavras-chave: Biometria; Diversidade genética; Pré-melhoramento.

\section{CHARACTERIZATION OF PEQUI FRUITS (Caryocar brasiliense Camb.) FROM BRAZILIAN CERRADO}

\begin{abstract}
The objectives of the present study were to characterize pequi fruits from Cerrado and to indicate some strategies of investigation, conservation and utilization of their genetic variability. Fruits were collected during two consecutive crop seasons (2007/2008 and 2008/2009) in eight Cerrado regions. Their physical characteristics were evaluated, and data were submitted to descriptive analysis, analysis of variance and simple correlations between characteristics. The results showed high phenotypic variability for most fruit characters in all sampled regions. Considering that some of this variability is heritable, there is a potential genetic gain by selection. The correlation analysis showed significant positive correlations among important traits for pequi breeding. A large difference among regions was observed about their potential to supply attractive fruits to the consumer market.
\end{abstract}

Keywords: Biometric; Genetic diversity; Pre-breeding.

\section{INTRODUÇÃO}

Dentre as espécies que se destacam no bioma Cerrado encontra-se o pequizeiro, Caryocar brasiliense Cambess, o qual é conhecido por seu valor econômico e nutricional, além da sua importância regional devido ao alto consumo de frutos e derivados pela população (CÔRREA et al., 2008, MOURA et al., 2013). Os frutos comercializados são, na sua grande maioria, provenientes de atividade extrativista. Devido à crescente devastação da vegetação nativa, a quantidade de plantas existentes vem diminuindo com o decorrer do tempo. Assim, a incorporação desta espécie aos sistemas produtivos regionais apresenta-se como uma alternativa bastante viável para a utilização racional dos recursos naturais

\footnotetext{
${ }^{1}$ Recebido em 26.06.2012 aceito para publicação em 03.09.2013

${ }^{2}$ Instituto Nacional de Pesquisas da Amazônia (INPA/MCTI). E-mail: <nara.moura@inpa.gov.com>.

${ }^{3}$ Universidade Federal de Goiás, Escola de Agronomia, Goiânia, GO, Brasil. E-mail: <lchaves@ufg.br> e < ronaldo@ufg.br>.
} 
do Cerrado, objetivando o desenvolvimento sustentável e a melhoria da qualidade de vida da população local.

Estudos morfológicos de frutos, sementes e desenvolvimento de plântulas e plantas jovens são frequentes para diversas espécies. Em geral são realizados visando auxiliar o conhecimento do sistema reprodutivo para auxiliar em programas de prémelhoramento de espécies não domesticadas. Abiometria de frutos e sementes, bem como o conhecimento da morfologia e desenvolvimento das plântulas, é fundamental para subsidiar estudos de germinação e produção de mudas auxiliando técnicas de cultivo para as espécies (BATTILANI et al, 2012). A existência de variabilidade em $C$. brasiliense para todos os caracteres de interesse, tanto os agronômicos, relacionados com a planta, como os de qualidade, relacionados com o fruto, permite esperar sucesso em um programa de melhoramento (OLIVEIRA et al., 2008).

Dentro deste contexto, o presente trabalho teve como objetivo caracterizar fisicamente frutos de pequizeiros provenientes do Cerrado para subsidiar estratégias de prospecção, preservação e utilização da variabilidade genética da espécie.

\section{MATERIAL E MÉTODOS}

Coletaram-se cinco frutos inteiros por planta de 76 matrizes de Caryocar brasiliense Camb. em oito grandes regiões do bioma Cerrado situadas nos estados de Goiás, Mato Grosso, Minas Gerais e Tocantins. Estas regiões foram definidas como: Região 1 - norte de Minas Gerais; Região 2 - nordeste de Goiás, região 3 - centro de Tocantins, região 4 - sul de Tocantins e norte de Goiás, região 5 - noroeste de Goiás, região 6 - médio Araguaia (GO e MT), região 7 - nordeste do Mato Grosso (MT) e região 8 - centro-oeste de Minas Gerais. A coleta foi realizada em duas safras consecutivas de produção, sendo as regiões 1 e 2 amostradas em 2007/2008 e as demais regiões em 2008/2009.

Os caracteres avaliados foram: massa do fruto (MF), diâmetro transversal do fruto (DTF), diâmetro longitudinal do fruto (DLF), número de pirênios por fruto (NP), massa total de pirênios (MTP), massa média de pirênios por fruto (MMP), média do diâmetro longitudinal de pirênios por fruto (DLP), média do diâmetro transversal de pirênios por fruto (DTP), rendimento de pirênios (REND) e massa de casca do fruto (MC). Os caracteres de massa foram obtidos com auxílio de uma balança digital e os resultados expressos em gramas. As medidas de dimensões foram obtidas com paquímetro digital e anotadas em milímetros. A massa média de pirênios por fruto (MMP) foi calculada a partir da massa total de pirênios por fruto e do número de pirênios por fruto (MTP/NP). O rendimento de pirênios (REND) foi obtido pela relação entre a massa total de pirênios e a massa total do fruto (MTP/MF). O caráter massa da casca (MC) foi obtido pela diferença entre a massa do fruto e a massa total de pirênios.

Os dados foram submetidos à estatística descritiva e, posteriormente, à análise de variância com base em modelo hierárquico que considera o efeito de regiões, matrizes dentro de regiões e frutos dentro de matrizes. Foram estimados os componentes de variância associados aos efeitos do modelo e as estimativas das proporções da variação fenotípica total que se deve a: diferença entre regiões $\left(P_{R}\right)$, diferença entre matrizes dentro regiões $\left(P_{M / R}\right)$ e diferença entre frutos dentro de matrizes dentro de regiões $\left(P_{F / M}\right)$. Também foram estimados os coeficientes de correlação fenotípica entre os caracteres avaliados. As análises foram realizadas com base em procedimento genéticoestatístico do software Genes (CRUZ, 1997).

\section{RESULTADOS}

A análise descritiva dos dez caracteres avaliados mostrou uma grande variação para os caracteres massa do fruto, diâmetro transversal do fruto, massa total de pirênios, massa média de pirênios por fruto e massa da casca por fruto (Tabela 1). Com base nos valores de coeficiente de variação fenotípica verificaram-se maiores variações para os caracteres massa total de pirênios (MTP) e massa da casca (MC), e menores para o número de pirênios por fruto (NP) e média do diâmetro longitudinal de pirênios por fruto (DLP).

As matrizes avaliadas de pequi possuem massa média de frutos variando de 22,6 g a 695,6 g (média de 180,9 g), massa total de pirênios variando de 6,6 g a 152,4 g (média de 41,8 g) e massa média de pirênios por fruto variando de 4,6 g a 112,2 g. O caráter MTP apresentou a maior variação fenotípica relativa com coeficiente de variação de 62,9\% (Tabela 1).

O número de pirênios variou de um a quatro com média de 1,7 pirênios por fruto. A média da massa de casca dos frutos foi de $139,1 \mathrm{~g}$. O rendimento de pirênios por fruto apresentou média de $23,5 \%$, o que significa que $76,5 \%$ da massa do fruto, em média, correspondem à casca. 
A análise de variância revelou a existência de variação significativa para quase todos os caracteres nos níveis estruturais analisados: regiões e matrizes dentro de regiões. A única exceção foi o caráter número de pirênios por fruto (NP) que apresentou variação significativa entre matrizes em apenas uma região (Tabela 2).

A maior parte da variação estimada encontra-se entre regiões, com poucas exceções. Dos caracteres com variação significativa entre matrizes, apenas o rendimento de pirênios por fruto (REND) apresentou uma maior proporção da variação total devido à diferença entre matrizes dentro de regiões (Tabela 3). O caráter diâmetro transversal do fruto (DTF) apresentou maior proporção da variância devida à diferença entre frutos dentro de matrizes dentro de região. Com base na decomposição da fonte de variação matriz dentro de regiões pôde-se verificar que as regiões que apresentaram maiores variabilidades para os caracteres físicos avaliados foram as regiões 2 (Norte de Minas Gerais), a região 06 (Noroeste de Goiás e a região 7 (médio Araguaia - GO e MT).

Tabela 1 - Valores médios, mínimos e máximos e coeficientes de variação fenotípica de caracteres físicos de frutos de Caryocar brasiliense provenientes de oito regiões do Cerrado.

Table 1 - Means, minimum and maximum values and phenotypic coefficient of variation of physical traits of Caryocar brasiliense fruits collected from eight Brazilian Cerrado regions.

\begin{tabular}{ccccccccccc}
\hline Plantas & MF & DTF & DLF & NP & DTP & DLP & MTP & MMP & REND & MC \\
\hline Média & 180,89 & 74,51 & 64,45 & 1,70 & 31,89 & 43,33 & 41,83 & 26,15 & 0,23 & 139,05 \\
Mínimo & 22,64 & 36,26 & 35,82 & 1,00 & 18,83 & 26,53 & 4,61 & 4,61 & 0,10 & 18,03 \\
Máximo & 695,53 & 129,86 & 104,79 & 4,00 & 49,52 & 70,96 & 152,37 & 112,18 & 0,47 & 553,11 \\
CV \% & 57,94 & 24,07 & 18,04 & 17,62 & 18,66 & 17,19 & 62,92 & 59,93 & 28,62 & 60,43 \\
\hline
\end{tabular}

MF: massa total do fruto (g), DTF: diâmetro transversal do fruto (mm), DLF: diâmetro longitudinal do fruto (mm), NP: número de pirênios por fruto, MS: massa total de pirênios (g) e MMP: massa média de pirênios por fruto (g), DTP: média de diâmetro transversal de pirênios por fruto (mm), DLP: média de diâmetro longitudinal de pirênios por fruto ( $\mathrm{mm})$, REND: rendimento de pirênios e MC: massa de casca do fruto (g).

Tabela 2 - Análise de variância de 10 caracteres físicos de frutos de Caryocar brasiliense provenientes de oito regiões do Cerrado.

Table 2 - Analysis of variance of 10 physical characteristics of Caryocar brasiliense fruits collected from eight Brazilian Cerrado regions.

\begin{tabular}{|c|c|c|c|c|c|c|c|c|c|c|c|}
\hline \multirow{2}{*}{$\begin{array}{c}\text { Fontes de } \\
\text { Variação }\end{array}$} & \multicolumn{5}{|l|}{ GL } & \multicolumn{6}{|c|}{ Quadrado Médio } \\
\hline & & $\mathrm{MF}$ & DTF & $\overline{D L F}$ & NP & DTP & DLP & MTP & MMP & REND & $\overline{M C}$ \\
\hline Matrizes & 75 & $38696^{* *}$ & $897,3^{* *}$ & $577,8^{* *}$ & $0,075^{N S}$ & $164,2^{* *}$ & $248,6^{* *}$ & $2254,9^{* *}$ & $1119,7^{* *}$ & $0,017 * *$ & $25821^{* *}$ \\
\hline Regiões & 7 & $227006 * *$ & $5732,2 * *$ & $4116,5^{* *}$ & $0,102^{\mathrm{NS}}$ & $1375,3^{* *}$ & $1790,0 * *$ & $16833,5 * *$ & $8851,8 * *$ & $0,070 * *$ & $137377 * *$ \\
\hline Mat (Regiões) & 68 & $193101 * *$ & $399,6 * *$ & $213,5^{* *}$ & $0,072^{\mathrm{NS}}$ & $39,5^{* *}$ & $90,0 * *$ & $754,2 * *$ & $323,7 * *$ & $0,011 * *$ & $14337 * *$ \\
\hline Mat (Reg. 01) & 29 & $15831 * *$ & $334,6 * *$ & $155,3 * *$ & $0,082^{\mathrm{NS}}$ & $27,3 * *$ & $39,4^{* *}$ & $543,7 *$ & $179,5^{* *}$ & $0,012 * *$ & $12647 * *$ \\
\hline Mat (Reg. 02) & 8 & $37925 * *$ & $860,4 * *$ & $344,9 * *$ & $0,087^{\mathrm{NS}}$ & $50,6 * *$ & $93,2 * *$ & $1570,3 * *$ & $310,3^{* *}$ & $0,006 * *$ & $27900 * *$ \\
\hline Mat (Reg. 03) & 5 & $8382^{\mathrm{NS}}$ & $215,6^{\mathrm{NS}}$ & $180,9 * *$ & $0,050^{\mathrm{NS}}$ & $49,5^{* *}$ & $85,4^{* *}$ & $504,5^{\mathrm{NS}}$ & $179,0 * *$ & $0,006 * *$ & $5177^{\mathrm{NS}}$ \\
\hline Mat (Reg. 04) & 10 & $1441^{\mathrm{NS}}$ & $100,9^{\mathrm{NS}}$ & $55,5 *$ & $0,057^{\mathrm{NS}}$ & $13,1^{* *}$ & $87,5^{* *}$ & $209,4^{\mathrm{NS}}$ & $49,1^{\mathrm{NS}}$ & $0,012 * *$ & $1141^{\mathrm{NS}}$ \\
\hline Mat (Reg. 05) & 7 & $6952^{\mathrm{NS}}$ & $241,6^{\mathrm{NS}}$ & $142,6 * *$ & $0,052^{\mathrm{NS}}$ & $16,6^{* *}$ & $95,4^{* *}$ & $151,7^{\mathrm{NS}}$ & $59,7^{\mathrm{NS}}$ & $0,011 * *$ & 5556* \\
\hline Mat (Reg. 06) & 4 & $109536 * *$ & $1308,5^{\text {NS }}$ & $557,6 * *$ & $0,056^{\mathrm{NS}}$ & $213,4 * *$ & $509,9 * *$ & $3494,8^{* *}$ & $2522,3^{* *}$ & $0,002^{\mathrm{NS}}$ & $74035^{* *}$ \\
\hline Mat (Reg. 07) & 1 & $607^{\mathrm{NS}}$ & $906,3^{* *}$ & $2045,8^{* *}$ & $0,318^{\mathrm{NS}}$ & $96,0^{* *}$ & $158,9 * *$ & $2966,3^{* *}$ & $2383,6 * *$ & $0,045 * *$ & $6258^{\mathrm{NS}}$ \\
\hline Mat (Reg. 08) & 4 & $1721^{\mathrm{NS}}$ & $167,0^{*}$ & $131,3^{* *}$ & $0,029 *$ & $11,9 *$ & $15,7 *$ & $83,1^{\text {NS }}$ & $12,0^{\mathrm{NS}}$ & $0,024 * *$ & $1593^{\mathrm{NS}}$ \\
\hline Resíduo & 304 & 4147 & 179,8 & 25,9 & 0,064 & 3,6 & 7,85 & 307,4 & 29,9 & 0,001 & 2434 \\
\hline CV (\%) & & 57,94 & 24,07 & 18,04 & 17,62 & 18,66 & 17,19 & 62,92 & 59,93 & 28,62 & 60,43 \\
\hline
\end{tabular}

MF: massa total do fruto (g), DTF: diâmetro transversal do fruto (mm), DLF: diâmetro longitudinal do fruto (mm), NP: número de pirênios por fruto, MS: massa total de pirênios (g) e MMP: massa média de pirênios por fruto (g), DTP: Média de diâmetro transversal de pirênios por fruto (mm), DLP: Média de diâmetro longitudinal de pirênios por fruto (mm), REND: relação massa de pirênios e massa de frutos e MC: massa de casca do fruto (g). CV\%: Coeficiente de variação residual; ${ }^{\text {Ns}}$ : não significativo; * e **Significativo a $5 \%$ e a $1 \%$ de probabilidade pelo teste $\mathrm{F}$, respectivamente. 
As médias dos caracteres de frutos por região confirmam a grande variação entre procedências (Tabela 3). A massa de frutos variou mais de cinco vezes da menor para a maior média enquanto a massa média de pirênios apresentou uma variação de nove vezes. As regiões com maiores médias são conhecidas por serem fornecedoras de frutos para o mercado, vindos do extrativismo. A região com frutos menores (Centro Oeste de MG) corresponde a uma população de pequizeiros anões, na região da Serra da Canastra.

Na análise de correlação entre todas as variáveis avaliadas constatou-se uma correlação positiva e significativa entre os caracteres massa total do fruto (MF), massa total de pirênios por fruto (MTP) e massa média de pirênios por fruto (MMP) (Tabela 4). Além disso, o caráter massa total do fruto (MF) mostrou-se altamente correlacionado com os demais caracteres dimensionais de frutos e pirênios (Tabela 4), exceto para número de pirênios (NP) e rendimento de pirênios (REND).

\section{DISCUSSÃO}

Os resultados demonstraram alta amplitude de variação para massa total dos frutos, o que pode ser devido à amostragem ampla, contemplando variadas regiões do Cerrado. Vera et al. (2007), avaliando caracteres de frutos em cinco regiões de Goiás, obtiveram média de massa de frutos de $125,1 \mathrm{~g}$, com intervalo de variação de 31,7 g a 496,1 g. Valores mais próximos foram encontrados por Luz et al. (2007) que estudaram a biometria de frutos e pirênios dessa espécie provenientes de três localidades do Norte de Minas Gerais e obtiveram intervalo de variação para massa de fruto de $22 \mathrm{~g}$ a 484 g. Gulias et al. (2008) avaliaram 15 árvores selecionadas no município de Damianópolis, GO, encontraram frutos com pesos médios variando de 126 g a 512 g, dentro do intervalo de variação encontrado no presente trabalho. Tais resultados demonstram que existem regiões do Cerrado que comportam uma ampla variabilidade fenotípica para caracteres de frutos, estas devem ser priorizadas, futuramente, em programa de conservação e melhoramento genético desta espécie.

O caráter massa total de pirênios (MTP) apresentou a maior variação relativa (4,6 g a 152,4 g). Vera et al. (2007) estudando duas regiões em Goiás, encontraram variação bem menor para esse caráter (médias de 11,5 g a 13,8 g), assim como Ramos \& Souza (2011) no Maranhão e Piauí. Gulias et al. (2008) encontraram médias de peso total de caroços variando de $44 \mathrm{~g}$ a 63 g. Tais resultados sugerem que para este caráter existe variação, portanto, estas informações poderão

Tabela 3 - Médias dos caracteres de frutos de Caryocar brasiliense por região e proporção da variância entre regiões $\left(\mathrm{P}_{\mathrm{R}}\right)$, entre matrizes dentro de regiões $\left(\mathrm{P}_{\text {Mat(reg) }}\right)$ e entre frutos dentro de matrizes $\left(\mathrm{P}_{\text {Frut(mat) }}\right)$.

Table 3 - Means of Caryocar brasiliense fruit characters by region and proportion of variance between regions $\left(P_{R}\right)$, plants within regions $\left(P_{\text {Mat (reg) }}\right)$ and fruits within plants $\left(P_{\text {Frut (mat })}\right)$.

\begin{tabular}{|c|c|c|c|c|c|c|c|c|c|c|}
\hline \multirow{2}{*}{ Região } & \multicolumn{10}{|c|}{ CARACTERES } \\
\hline & MF & DTF & DLF & NP & DTP & DLP & MTP & MMP & REND & MC \\
\hline Norte de MG (1) & 204,88 & 78,81 & 68,63 & 1,67 & 35,09 & 46,50 & 48,69 & 30,73 & 0,24 & 156,20 \\
\hline Nordeste de GO (2) & 248,06 & 84,71 & 70,25 & 1,71 & 33,31 & 43,81 & 43,43 & 25,60 & 0,18 & 204,64 \\
\hline Centro do TO (3) & 138,89 & 69,37 & 57,64 & 1,87 & 30,13 & 42,76 & 37,41 & 21,55 & 0,27 & 101,49 \\
\hline Sul do TO e Norte de GO (4) & 120,10 & 68,42 & 56,81 & 1,96 & 26,91 & 38,57 & 29,48 & 15,63 & 0,24 & 90,62 \\
\hline Noroeste de Goiás (5) & 110,18 & 61,98 & 56,27 & 1,63 & 24,85 & 37,25 & 20,75 & 13,23 & 0,19 & 89,44 \\
\hline Médio Araguaia GO e MT (6) & 291,23 & 87,83 & 78,16 & 1,56 & 38,39 & 48,97 & 63,99 & 44,30 & 0,23 & 227,23 \\
\hline Nordeste de MT (7) & 300,57 & 92,77 & 83,74 & 1,47 & 44,29 & 62,55 & 113,43 & 80,77 & 0,38 & 187,14 \\
\hline Centro Oeste MG (8) & 55,04 & 49,39 & 45,50 & 1,48 & 23,01 & 31,03 & 13,28 & 9,01 & 0,25 & 41,76 \\
\hline$P_{R}$ & 40,51 & 35,94 & 59,16 & 1,02 & 74,41 & 62,25 & 48,82 & 69,36 & 28,57 & 37,57 \\
\hline$P_{\text {Mat(reg) }}$ & 25,13 & 12,59 & 24,17 & 2,51 & 17,00 & 25,54 & 11,53 & 20,30 & 41,82 & 30,87 \\
\hline$P_{\text {Frut(mat })}$ & 34,36 & 51,47 & 16,67 & 96,47 & 8,57 & 12,21 & 39,65 & 10,34 & 29,61 & 31,56 \\
\hline
\end{tabular}

MF: massa total do fruto (g), DTF: diâmetro transversal do fruto (mm), DLF: diâmetro longitudinal do fruto (mm), NP: número de pirênios por fruto, MS: massa total de pirênios (g) e MMP: massa média de pirênios por fruto (g), DTP: Média de diâmetro transversal de pirênios por fruto (mm), DLP: Média de diâmetro longitudinal de pirênios por fruto (mm), REND: relação massa de pirênios por massa de frutos e MC: massa de casca do fruto $(\mathrm{g}) . P_{R}$ : proporção da variância total que se deve à diferença entre regiões; $\mathrm{P}_{\text {Mç }}$ : proporção da variância total que se deve à diferença entre matrizes dentro de uma mesma região e $\mathrm{P}_{\mathrm{F} \text { (Mat) }}$ : proporção da variância total que se deve à diferença entre frutos dentro de matrizes.

Revista Árvore, Viçosa-MG, v.37, n.5, p.905-912, 2013 
Tabela 4 - Estimativas de coeficientes de correlação fenotípica (abaixo da diagonal) e residual (acima da diagonal) entre variáveis de 76 plantas de Caryocar brasiliense.

Table 4 - Estimate of phenotypic (below diagonal) and environmental (above diagonal) correlation coefficients among variables of 76 Caryocar brasiliense plants.

\begin{tabular}{|c|c|c|c|c|c|c|c|c|c|c|}
\hline & MF & DTF & DLF & NP & DTMP & DLMP & MTP & MMP & REND & MC \\
\hline MF & - & $0,85 * *$ & $0,58 * *$ & $0,74 * *$ & $0,24 * *$ & $0,17 * *$ & $0,90 * *$ & $0,088 \mathrm{NS}$ & $0,21 * *$ & $0,99 * *$ \\
\hline DTF & $0,94 * *$ & - & $0,51 * *$ & $0,84 * *$ & $0,23 * *$ & $0,11 *$ & $0,82 * *$ & $-0,04 \mathrm{NS}$ & $0,41 * *$ & $0,82 * *$ \\
\hline DLF & $0,88 * *$ & $0,82 * *$ & - & $0,38 * *$ & $0,67 * *$ & $0,66^{* *}$ & $0,47 * *$ & $0,50 * *$ & $0,14 * *$ & $0,58 * *$ \\
\hline NP & $0,10 \mathrm{NS}$ & $0,29 * *$ & $0,08 \mathrm{NS}$ & - & $0,05 \mathrm{NS}$ & $-0,06 \mathrm{NS}$ & $0,84 * *$ & $-0,26 * *$ & $0,62 * *$ & $0,66 * *$ \\
\hline DTMP & $0,80 * *$ & $0,77 * *$ & $0,87 * *$ & $-0,16 * *$ & - & $0,86 * *$ & $0,19 * *$ & $0,73 * *$ & $0,18 * *$ & $0,25 * *$ \\
\hline DLMP & $0,68 * *$ & $0,66 * *$ & $0,81 * *$ & $-0,20 * *$ & $0,88 * *$ & - & $0,10 * *$ & $0,81 * *$ & $0,14 * *$ & $0,18 * *$ \\
\hline МТP & $0,81 * *$ & $0,84 * *$ & $0,75 * *$ & $0,19 * *$ & $0,85 * *$ & $0,80 * *$ & - & $0,03 \mathrm{NS}$ & $0,54 * *$ & $0,81 * *$ \\
\hline MMP & $0,74 * *$ & $0,66 * *$ & $0,80 * *$ & $-0,25 * *$ & $0,91 * *$ & $0,90 * *$ & $0,86 * *$ & - & $0,01 \mathrm{NS}$ & $0,10^{*}$ \\
\hline REND & $-0,19 * *$ & $-0,07 \mathrm{NS}$ & $-0,16 * *$ & $0,23 * *$ & $0,17 * *$ & $0,24 * *$ & $0,37 * *$ & $0,24 * *$ & - & $0,09 \mathrm{NS}$ \\
\hline MC & $0,98 * *$ & $0,91 * *$ & $0,86 * *$ & $0,06 \mathrm{NS}$ & $0,73 * *$ & $0,60 * *$ & $0,70 * *$ & $0,65 * *$ & $-0,34 * *$ & - \\
\hline
\end{tabular}

MF: massa total do fruto (g), DTF: diâmetro transversal do fruto (mm), DLF: diâmetro longitudinal do fruto (mm), NP: número de pirênios por fruto, MS: massa total de pirênios (g) e MMP: massa média de pirênios por fruto (g), DTP: Média de diâmetro transversal de pirênios por fruto (mm), DLP: Média de diâmetro longitudinal de pirênios por fruto (mm), REND: relação massa de pirênios por massa de frutos e MC: massa de casca do fruto (g). ${ }^{\text {Ns}}$ : não significativo; * e **Significativo a $5 \%$ e a $1 \%$ de probabilidade pelo teste t, respectivamente.

nortear as futuras coletas de sementes para diferentes fins, principalmente, para as matrizes que encontram-se georeferenciadas.

A grande amplitude de variação encontrada nos caracteres diâmetro longitudinal do fruto (DTF) e diâmetro transversal do fruto (DTF) coincidem com os resultados obtidos por Luz et al. (2007). No entanto, Vera et al. (2005, 2007) encontraram valores bem inferiores aos resultados obtidos no presente trabalho. Luz et al. (2007) destacam a grande variação encontrada entre localidades para os caracteres comprimento, diâmetro e massa do fruto. Este resultado sugere que os frutos de pequizeiro são diferentes em massa e volume entre locais de coleta.

O número de pirênios está de acordo com os resultados encontrados por Vera et al. (2005, 2007), Luz et al. (2007) e Corrêa et al. (2008). Vera et al. (2007) citam que o número de pirênios por frutos encontrados em diversos trabalhos varia de um a três e, que, é nítida predominância da ocorrência de frutos com apenas um pirênio, o que corrobora com os resultados obtidos neste trabalho. O caráter número de pirênios apresentou um coeficiente de variação relativamente baixo em relação aos outros caracteres e foi próximo ao encontrado por Oliveira et al. (2009) em outra espécie (C. coriaceum Wittm) encontrada na região norte do país.

A alta proporção de casca encontrada no presente trabalho e, consequentemente, baixo rendimento de pirênios por fruto são características indesejáveis para a exploração econômica do pequi, significando que a melhoria de tal característica deverá ser priorizada em programa de melhoramento. Para um programa de melhoramento da espécie seria aconselhável a seleção de matrizes que apresentam maiores números de pirênios e menor massa da casca, consequentemente maior rendimento de pirênios por fruto. A região onde se verificou o maior valor médio para rendimento de pirênios foi a região 8 (Nordeste do $M T$ ), destacando-se a matriz 71, com frutos que apresentaram média de massa total do fruto de 292,8 g, média de massa total de pirênios de 130,7 g e massa da casca de $162,0 \mathrm{~g}$.

De maneira geral, a variação encontrada para todos os outros caracteres avaliados são semelhantes aos obtidos por Vera et al. (2005), confirmando a quantidade de variação existente nas populações de Caryocar brasiliense a ser explorada em programas de melhoramento e domesticação da espécie, principalmente para o caráter massa de frutos, que apresentou variação expressiva inclusive entre frutos de uma mesma árvore. Durante, o período de coleta dos frutos, também foram observadas diferenças marcantes quanto ao grau de amadurecimento dos frutos. Assim, é comum em algumas regiões e até mesmo dentro de regiões encontrarem-se frutos em vários estágios de maturação. Segundo Vera et al. (2005), tais diferenças podem resultar em diferenças na massa e volume dos frutos.

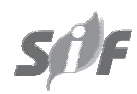

Revista Árvore, Viçosa-MG, v.37, n.5, p.905-912, 2013 
Na análise de variância dos caracteres físicos deve-se ressaltar que a fonte de variação regiões apresenta um confundimento de efeitos pelo fato de terem sido coletadas em duas safras. Observações de campo realizadas em 15 áreas de cinco regiões de Goiás permitem afirmar que existe uma boa repetibilidade de caracteres de frutos entre anos, considerando-se plantas individuais (FERREIRA, 2007). Acredita-se, portanto, que a influência de regiões seja maior que a influência do efeito de anos sobre os caracteres de frutos. De maneira geral, a variação encontrada para todos os outros caracteres avaliados indica a quantidade de variação existente nas populações estudadas de pequi a ser explorada em programas de melhoramento e domesticação da espécie, principalmente para o caráter massa de frutos, que apresentou variação expressiva inclusive entre frutos de uma mesma árvore.

A variação fenotípica existente em plantas nativas do Cerrado é bastante influenciada por componentes ambientais não controlados, como a condição de antropização, o solo, o clima, a idade das plantas e também pelas próprias diferenças genéticas entre os indivíduos. Se for considerado que parte dessa variabilidade seja de natureza genética, vislumbra-se a possibilidade de seleção daquelas plantas que produzam frutos com os melhores atributos de qualidade para o consumidor (GANGA et al., 2009).

Os resultados do presente trabalho confirmam que a proporção de variação fenotípica para caracteres de frutos entre grandes regiões é muito expressiva e deve ser explorada, tanto para fins comerciais quanto conservacionistas ou de manejo de recursos genéticos. Em outros trabalhos, onde a proposta foi avaliar a variação entre diferentes regiões situadas no estado de Goiás, observaram-se maiores diferenças entre frutos na planta e entre árvores (VERA et al., 2005).

Os valores médios dos caracteres (Tabela 3) mostram que algumas regiões se destacam pelo seu potencial quanto ao tamanho de frutos e de pirênios. Os maiores valores foram encontrados nas populações das regiões do vale do Araguaia e Nordeste de Mato Grosso. Essas regiões são contíguas ocorrendo plantas com frutos de grande tamanho. Algumas destas plantas foram coletadas neste estudo. Há uma hipótese corrente na região que estes pequizeiros teriam sofrido um início de domesticação pelos índios Caiapós, que atualmente cultivam pequenas roças da espécie no Parque Indígena do Xingu, vizinho da região. Esta hipótese merece um estudo à parte para ser testada.
O conhecimento prévio das relações existentes entre caracteres de frutos auxiliam na orientação de estratégia de seleção a ser adotada para obtenção de genótipos mais produtivos para diversos fins, como: maior número de pirênios, massa total de frutos ou massa média de pirênios. A correlação existente entre os caracteres permite uma orientação na seleção, quando se objetiva o aprimoramento dos genótipos para um conjunto de caracteres e não para cada um de forma isolada, tornando possível a seleção indireta de caracteres desejáveis correlacionados positivamente (VENCOVSKY; BARRIGA, 1992). Apesar de serem correlações fenotípicas, caracteres que apresentam valores elevados (positivos ou negativos) possivelmente deverão apresentar também correlações genéticas significativas.

Correlações significativas e elevadas encontradas entre massa do fruto e os caracteres dimensionais de frutos e pirênios (como por exemplo, o diâmetro transversal do fruto) são desejadas para o melhoramento da espécie, pois selecionando matrizes com frutos que apresentam diâmetros transversais maiores, consequentemente, estaria selecionando maior número de pirênios por fruto, o que poderia elevar o rendimento de pirênios por fruto. Quando as correlações são positivas e de alta magnitude, os caracteres podem ser considerados uma única unidade de seleção. Por sua vez, as correlações desfavoráveis geralmente dificultam a seleção simultânea dos caracteres superiores nos programas de melhoramento. A correlação desfavorável e altamente significativa encontrada entre massa do fruto e massa da casca exerce uma maior influência no rendimento de pirênios por fruto. Vera et al. (2007) encontraram uma relação constante entre massa da casca e massa do fruto, e citam que esta é uma característica indesejável, pois a proporção alta de casca no fruto de pequi, atualmente, representa um sério problema ambiental para as pequenas agroindústrias processadoras de pequi. Assim, esta alta correlação seria um fator limitador para o melhoramento de plantas.

Quando se avaliam as correlações com o caráter rendimento de pirênios por fruto (REND) fica evidenciado que os caracteres que são positivamente correlacionados são aqueles relacionados a medidas tomadas em pirênios, consequentemente, para um programa de melhoramento da espécie seria aconselhável a seleção de matrizes que apresentam maiores números de pirênios e menor massa da casca. Corrêa et al. (2008) citam que essa característica representa uma limitação em futuros trabalhos de melhoramento, tendo-se em vista que o número de pirênios 
é de fundamental importância na exploração econômica do pequi. Por outro lado, a correlação positiva entre massa de fruto e massa de pirênios favorece o melhoramento da espécie, pois a seleção de plantas que possuam frutos com maior peso, caráter que a sua avaliação é menos onerosa, favorece o aumento de massas de pirênios e, possivelmente, o rendimento para agroindústria será elevado. Outro caráter de fácil identificação visual é o número de pirênios por fruto por meio da observação de lóculos no fruto. Assim, a correlação baixa, porém positiva entre o caráter número de pirênios e rendimento deverá facilitar o trabalho de melhoristas, pois selecionando matrizes com número elevado de pirênios por fruto possivelmente aumentará o rendimento.

\section{CONCLUSÕES}

Os resultados deste trabalho evidenciaram uma elevada variabilidade fenotípica para a maioria dos caracteres físicos de frutos de pequizeiro nas regiões amostradas. Considerando que parte dessa variabilidade seja de natureza genética, isto indica potencial de ganho genético por meio da seleção. Existe uma grande diferença entre regiões quanto ao potencial de fornecimento de frutos adequados ao mercado.

\section{AGRADECIMENTOS}

Á FINEP (Financiadora de Estudos e Projetos) pelo apoio financeiro, ao Professor J. L. do Nascimento pela ajuda na coleta de campo e a Dra. Fabiane Rabelo da Costa pelas sugestões feitas para a elaboração deste artigo. À CAPES (Coordenação de Aperfeiçoamento de Pessoal de Nível Superior - MEC - Brasil) pela bolsa de doutorado e ao CNPq (Conselho Nacional de Desenvolvimento Científico e Tecnológico) pela bolsa de produtividade em pesquisa.

\section{REFERÊNCIAS}

BATTILANI, J. L.; SANTIAGO, E. F.; DIAS, E. S. Morfologia de frutos, sementes, plântulas e plantas jovens de Guibourtia hymenifolia (Moric.) J. Leonard (Fabaceae). Revista Árvore, v.35, n.5, p.1089-1098, 2011.

CÔRREA, G. C. et al. Determinações físicas em frutos e sementes de baru (Dipteryx alata Vog.), cajuzinho (Anacardium othonianum Rizz.) e pequi (Caryocar brasiliense Camb.), visando melhoramento genético. Bioscience Journal, v.24, n.4, p.42-47, 2008.
Ferreira, G. A. Produção de frutos e entomofauna associada a pequizeiro (Caryocar brasiliense Camb.) no Cerrado do Estado de Goiás. 2007. Tese (Doutorado em Agronomia) - Universidade Federal de Goiás, Goiânia, 2007.

GANGA, R. M. D.; CHAVES, L. C.; NAVES, R. V. Parâmetros genéticos em progênies de Hancornia speciosa Gomes do Cerrado. Scientia Forestalis, v.37, n.84, p.395-404, 2009.

GULIAS, A. P. S. M.; RIBEIRO, J. F.; OLIVEIRA, M. C. DE; AQUINO, F. G.; SILVA, M. R. DA. Produtividade dos Pequizeiros (Caryocar brasiliense Camb.) no município de Damianópolis, Goiás. Anais... IX simpósio Nacional dos Cerrados e II Simpósio Internacional Savanas Tropicais. Desafios e estratégias para o equilíbrio entre sociedade, agronegócio e recursos naturais. 2008.

LUZ, G. R. et al. Biometria de frutos e taxa de ataque por insetos a frutos e putâmens de Caryocar brasiliense Camb. (caryocaraceae) provenientes de três localidades do norte de minas gerais. In: CONGRESSO DE ECOLOGIA DO BRASIL, 8., 2007, Caxambú. Anais... Caxambú: 2007.

MOURA, N. F. et al. Variabilidade entre procedências e progênies de Pequizero (Caryocar brasiliense Camb.). Revista Scientia Forestalis, v.41, n.97, p.103-112, 2013.

OLIVEIRA, M. E. B. et al. Caracterização física de frutos do pequizeiro nativos da chapada do araripe. Revista Brasileira de Fruticultura, v.31, n.4, p.1196-1201, 2009.

RAMOS, K. M. C.; SOUZA, V. A. B. Características físicas e químico-nutricionais de frutos de pequizeiro (Caryocar coriaceum WITTM.) em populações naturais da região meionorte do brasil. Revista Brasileira de Fruticultura, v.33, n.2, p.500-508, 2011.

VENCOVSKY, R.; BARRIGA, P. Genética biométrica no fitomelhoramento. Ribeirão Preto: Sociedade Brasileira de Genética, 1992. 496p.

Revista Árvore, Viçosa-MG, v.37, n.5, p.905-912, 2013 
VERA, R. et al. Caracterização física de frutos do pequizeiro (Caryocar brasiliense Camb.) no Estado de Goiás. Pesquisa Agropecuária Tropical, v.35, n.1, p.7179, 2005.
VERA, R. et al. Caracterização física e química de frutos do pequizeiro (Caryocar brasiliense Camb.) oriundos de duas regiões no estado de Goiás, Brasil. Pesquisa Agropecuária Tropical, v.37, n.1, p.93-99, 2007. 